\title{
The Effect of Hospitalist Discontinuity on Adverse Events
}

\author{
Kevin J. O'Leary, MD, MS ${ }^{1 \star}$, Jonathan Turner, $\mathrm{PhD}^{2}$, Nicholas Christensen, BA ${ }^{1}$, Madeleine Ma, MS³, Jungwha Lee, $\mathrm{PhD}$, MPH${ }^{3}$, \\ Mark V. Williams, $\mathrm{MD}^{4}$, Luke O. Hansen, MD, MHS ${ }^{1}$
}

\begin{abstract}
'Division of Hospital Medicine, Northwestern University Feinberg School of Medicine, Chicago, Illinois; '2Department of Systems Engineering, University Hospital, Augusta, Georgia; ${ }^{3}$ Biostatistics Collaboration Center, Department of Preventive Medicine, Northwestern University Feinberg School of Medicine, Chicago, Illinois; ${ }^{4}$ Center for Health Services Research, University of Kentucky, Lexington, Kentucky.
\end{abstract}

BACKGROUND: Patient-physician continuity is difficult to achieve in hospital settings because of the need to provide care continuously. The impact of hospital physician discontinuity on patient safety is unknown.

OBJECTIVE: To determine the association between hospital physician continuity and the incidence of adverse events (AEs).

DESIGN: Retrospective observational study using multivariable models to adjust for patient characteristics.

PARTICIPANTS: Patients admitted to a nonteaching hospitalist service in a large academic hospital between March 1, 2009 and December 31, 2011.

MAIN MEASURE(S): Two measures of continuity were used. The Number of Physicians Index (NPI) was the total number of unique hospitalists caring for a patient. The Usual Provider of Care (UPC) Index was the proportion of encounters with the most frequently encountered hospitalist. Outcome measures were AEs detected by automated queries of information systems and confirmed by 2 physician researchers.

KEY RESULTS: Our analysis included data from 474 hospitalizations. In unadjusted models, each 1-unit increase in the NPI (ie, less continuity) was significantly associated with the incidence of 1 or more AEs (odds ratio $=1.75$; $P<0.001)$. However, UPC was not associated with incidence of AEs. Across all adjusted models, neither NPI nor UPC was significantly associated with the incidence of AEs. The direction of the effect of discontinuity on AEs was also inconsistent across models.

CONCLUSIONS: Hospitalist physician continuity does not appear to be associated with the incidence of AEs. Because hospital care is provided by teams of clinicians, future research should evaluate the impact of team complexity and dynamics on patient outcomes. Journal of Hospital Medicine 2015;10:147-151. (c) 2014 Society of Hospital Medicine
Although definitions vary, continuity of care can be thought of as the patient's experience of a "continuous caring relationship" with an identified healthcare professional. ${ }^{1}$ Research in ambulatory settings has found that patients who see their primary care physician for a higher proportion of office visits have higher patient satisfaction, better hypertensive control, lower risk of hospitalization, and fewer emergency department visits. ${ }^{2-5}$ Continuity with a single hospital-based physician is difficult to achieve because of the need to provide care 24 hours a day, 7 days a week. Key clinical information may be lost during physician-to-physician handoffs (eg, at admission, at the end of rotations on service) during hospitalization. Our research group recently found that lower hospital physician continuity was associated with modestly increased hospital costs, but also a trend toward lower

*Address for correspondence and reprint requests: Kevin J. O'Leary, MD, Associate Professor of Medicine, Division of Hospital Medicine, Northwestern University Feinberg School of Medicine, Chicago, IL 60611; Telephone: 312-926-5924; Fax: 312-926-4588; E-mail: keoleary@nmh.org

Additional Supporting Information may be found in the online version of this article.

Received: September 29, 2014; Revised: November 18, 2014; Accepted: November 30, 2014

2014 Society of Hospital Medicine DOI 10.1002/jhm.2308

Published online in Wiley Online Library (Wileyonlinelibrary.com). readmissions. ${ }^{6}$ We speculated that physicians newly taking over patient care from colleagues reassess diagnoses and treatment plans. This reassessment may identify errors missed by the previous hospital physician. Thus, discontinuity may theoretically help or hinder the provision of safe hospital care.

We sought to examine the relationship between hospital physician continuity and the incidence of adverse events (AEs). We combined data from 2 previously published studies by our research group; one investigated the relationship between hospital physician continuity and costs and 30-day readmissions, the other assessed the impact of unit-based interventions on AEs. ${ }^{6,7}$

\section{METHODS}

\section{Setting and Study Design}

This retrospective, observational study was conducted at Northwestern Memorial Hospital, an 876-bed tertiary care teaching hospital in Chicago, Illinois, and was approved by the institutional review board of Northwestern University. Subjects included patients admitted to an adult nonteaching hospitalist service between March 1, 2009 and December 31, 2011. Hospitalists on this service worked without resident physicians in rotations usually lasting 7 consecutive days beginning on Mondays and ending on Sundays. Hospitalists were allowed to switch portions of their 
schedule with one another, creating the possibility that certain rotations may have been slightly shorter or longer than 7 days. Hospitalists gave verbal signout via telephone to the hospitalist taking over their service on the afternoon of the last day of their rotation. These handoffs customarily involved both hospitalists viewing the electronic health record during the discussion but were not standardized. Night hospitalists performed admissions and cross-coverage each night from 7 PM to 7 AM. Night hospitalists printed history and physicals for day hospitalists, but typically did not give verbal sign-out on new admissions.

\section{Acquisition of Study Population Data}

We identified all patients admitted to the nonteaching hospitalist service using the Northwestern Medicine Enterprise Data Warehouse (EDW), an integrated repository of all clinical and research data sources on the campus. We excluded patients admitted under observation status, those initially admitted to other services (eg, intensive care, general surgery), those discharged from other services, and those cared for by advanced practice providers (ie, nurse practitioners and physician assistants).

\section{Predictor Variables}

We identified physicians completing the primary service history and physicals (H\&P) and progress notes throughout patients' hospitalizations to calculate 2 measures of continuity: the Number of Physicians Index (NPI), and the Usual Provider of Continuity (UPC) Index. ${ }^{8,9}$ The NPI represented the total number of unique hospitalists completing H\&Ps and/or progress notes for a patient. The UPC was calculated as the largest number of notes signed by a single hospitalist divided by the total number of hospitalist notes for a patient. For example, if Dr. John Smith wrote notes on the first 4 days of a patient's hospital stay, and Dr. Mary Jones wrote notes on the following 2 days (total stay $=6$ days), the NPI would be 2 and the UPC would be 0.67 . Therefore, higher NPI and lower UPC designate lower continuity. Significant events occurring during the nighttime were documented in separate notes titled cross-cover notes. These crosscover notes were not included in the calculation of NPI or UPC. In the rare event that 2 or more progress notes were written on the same day, we selected the one used for billing to calculate UPC and NPI.

\section{Outcome Variables}

We used AE data from a study we conducted to assess the impact of unit-based interventions to improve teamwork and patient safety, the methods of which have been previously described. ${ }^{7}$ Briefly, we used a 2stage medical record review similar to that performed in prior studies. ${ }^{10-13}$ In the first stage, we identified potential AEs using automated queries of the Northwestern Medicine EDW. These queries were based on screening criteria used in the Harvard Medical Practice Study and the Institute for Healthcare Improvement (IHI) Global Trigger Tool. ${ }^{12,13}$ Examples of queries included abnormal laboratory values (eg, international normalized ratio [INR] $>6$ after hospital day 2 and excluding patients with INR $>4$ on day 1 ), administration of rescue medications (eg, naloxone), certain types of incident reports (eg, pressure ulcer), International Classification of Diseases, Ninth Revision (ICD-9) codes indicating hospital-acquired conditions (eg, venous thromboembolism), and text searches of progress notes and discharge summaries using natural language processing. ${ }^{14}$ Prior research by our group confirmed these automated screens identify a similar number of AEs as manual medical record screening. ${ }^{14}$ For each patient with 1 or more potential $\mathrm{AE}$, a research nurse performed a medical record abstraction and created a description of each potential AE.

In the second stage, 2 physician researchers independently reviewed each potential $\mathrm{AE}$ in a blinded fashion to determine whether or not an AE was present. An AE was defined as injury due to medical management rather than the natural history of the illness, ${ }^{15}$ and included injuries that prolonged the hospital stay or produced disability as well as those resulting in transient disability or abnormal lab values. ${ }^{16}$ After independent review, physician reviewers discussed discrepancies in their ratings to achieve consensus.

We tested the reliability of medical record abstractions in our prior study by conducting duplicate abstractions and consensus ratings for a randomly selected sample of 294 patients. ${ }^{7}$ The inter-rater reliability was good for determining the presence of AEs $(\kappa=0.63)$.

\section{Statistical Analyses}

We calculated descriptive statistics for patient characteristics. Primary discharge diagnosis ICD-9 codes were categorized using the Healthcare Cost and Utilization Project Clinical Classification Software. ${ }^{17} \mathrm{We}$ created multivariable logistic regression models with the independent variable being the measure of continuity (NPI or UPC) and the dependent variable being experiencing 1 or more AEs. Covariates included patient age, sex, race, payer, night admission, weekend admission, intensive care unit stay, Medicare Severity Diagnosis Related Group (MS-DRG) weight, and total number of Elixhauser comorbidities. ${ }^{18}$ The length of stay (LOS) was also included as a covariate, as longer LOS increases the probability of discontinuity and may increase the risk for AEs. Because MSDRG weight and LOS were highly correlated, we created several models; the first including both as continuous variables, the second including both categorized into quartiles, and a third excluding MS-DRG weight and including LOS as a continuous variable. Our 


\begin{tabular}{lc} 
TABLE 1. Patient and Hospitalization & \\
Characteristics (N=474) & \\
\hline Characteristic & Value \\
\hline Mean age (SD), y & $55.1(18.8)$ \\
Mean length of stay (SD), d & $3.4(3.1)$ \\
Women, $n$ (\%) & $241(50.8)$ \\
Nonwhite race, $n$ (\%) & $233(49.2)$ \\
Payer, $n$ (\%) & \\
Private & $180(38)$ \\
Medicare & $165(34.8)$ \\
Medicaid & $47(9.9)$ \\
Self-pay/other & $82(17.3)$ \\
Night admission, $n$ (\%) & $245(51.7)$ \\
Weekend admission, $n$ (\%) & $135(28.5)$ \\
Intensive care unit stay, $n$ (\%) & $18(3.8)$ \\
Diagnosis, $n$ (\%) & \\
Diseases of the circulatory system & $95(20.0)$ \\
Diseases of the digestive system & $65(13.7)$ \\
Diseases of the respiratory system & $49(10.3)$ \\
Injury and poisoning & $41(8.7)$ \\
Diseases of the skin and soft tissue & $31(6.5)$ \\
Symptoms, signs, and ill-defined conditions and factors influencing health status & $28(5.9)$ \\
Endocrine, nutritional, and metabolic diseases and immunity disorders & $25(5.3)$ \\
Diseases of the genitourinary system & $24(5.1)$ \\
Diseases of the musculoskeletal system and connective tissue & $23(4.9)$ \\
Diseases of the nervous system & $23(4.9)$ \\
Other & $70(14.8)$ \\
Mean no. of Elixhauser comorbidities (SD) & $2.3(1.7)$ \\
Mean MS-DRG weight (SD) & $1.0(1.0)$ \\
Mean NPI (SD) & $2.5(1.0)$ \\
Mean UPC (SD) & $0.6(0.2)$ \\
& \\
&
\end{tabular}

NOTE: Abbreviations: MS-DRG, Medicare severity diagnosis-related group; NPI, Number of Physicians Index; SD, standard deviation; UPC, Usual Provider of Care Index.

prior study assessing the impact of unit-based interventions did not show a statistically significant difference in the pre- versus postintervention period, thus we did not include study period as a covariate.

\section{RESULTS}

\section{Patient Characteristics}

Our analyses included data from 474 hospitalizations. Patient characteristics are shown in Table 1. Patients were a mean $51.1 \pm 18.8$ years of age, hospitalized for a mean $3.4 \pm 3.1$ days, included $241(50.8 \%)$ women, and $233(49.2 \%)$ persons of nonwhite race. The mean and standard deviation of NPI and UPC were $2.5 \pm 1.0$ and $0.6 \pm 0.2$. Overall, 47 patients $(9.9 \%)$ experienced 55 total AEs. AEs included 31 adverse drug events, 6 falls, 5 procedural injuries, 4 manifestations of poor glycemic control, 3 hospital-acquired infections, 2 episodes of acute renal failure, 1 episode of delirium, 1 pressure ulcer, and 2 categorized as other.

\section{Association Between Continuity and Adverse Events}

In unadjusted models, each 1-unit increase in the NPI (ie, less continuity) was significantly associated with the incidence of 1 or more AEs (odds ratio
TABLE 2. Effect of Decreased Continuity on Adverse Events

\begin{tabular}{lcccc}
\hline & $\begin{array}{c}\text { NPI OR } \\
(95 \% \mathrm{Cl})^{\star}\end{array}$ & $\begin{array}{c}P \\
\text { Value }\end{array}$ & $\begin{array}{c}\text { UPC OR } \\
(95 \% \mathrm{Cl})^{*}\end{array}$ & $\begin{array}{c}P \\
\text { Value }\end{array}$ \\
\hline $\begin{array}{l}\text { Unadjusted model } \\
\text { Adjusted models }\end{array}$ & $1.75(1.33-2.29)$ & $<0.0001$ & $1.03(0.89-1.21)$ & 0.68 \\
Model 1 MS-DRG and LOS continuous & $1.16(0.78-1.72)$ & 0.47 & $0.96(0.79-1.14)$ & 0.60 \\
Model 2 MS-DRG and LOS in quartiles & $1.38(0.98-1.94)$ & 0.07 & $1.05(0.88-1.26)$ & 0.59 \\
Model 3 MS-DRG dropped, & $1.14(0.77-1.70)$ & 0.51 & $0.95(0.79-1.14)$ & 0.56 \\
LOS continuous & & & & \\
\hline
\end{tabular}

NOTE: Abbreviations: Cl, confidence interval; LOS, length of stay; MS-DRG, Medicare severity diagnosisrelated group; NPI, Number of Physicians Index; OR, odds ratio; UPC, Usual Provider Of Continuity Index. ${ }^{*} \mathrm{NPI}$ is the total number of unique hospitalist physicians. UPC is the largest number of encounters by a single hospitalist physician divided by the total number of hospitalist physician encounters for a patient. The OR for UPC reflects a $10 \%$ decrease.

$[\mathrm{OR}]=1.75 ; P<0.001)$. However, UPC was not associated with incidence of AEs $(\mathrm{OR}=1.03 ; P=0.68)$ (Table 2). Across all adjusted models, neither NPI nor UPC was significantly associated with the incidence of AEs. The direction of the effect of discontinuity on AEs was inconsistent across models. Though all 3 adjusted models using NPI as the independent variable showed a trend toward increased odds of experiencing 1 or more $\mathrm{AE}$ with discontinuity, 2 of the 3 models using UPC showed trends in the opposite direction.

\section{DISCUSSION}

We found that hospitalist physician continuity was not associated with the incidence of AEs. Our findings are somewhat surprising because of the high value placed on continuity of care and patient safety concerns related to handoffs. Key clinical information may be lost when patient care is transitioned to a new hospitalist shortly after admission (eg, from a night hospitalist) or at the end of a rotation. Thus, it is logical to assume that discontinuity inherently increases the risk for harm. On the other hand, a physician newly taking over patient care from another may not be anchored to the initial diagnosis and treatment plan established by the first. This "second look" could potentially prevent missed/delayed diagnoses and optimize the plan of care. ${ }^{19}$ These countervailing forces may explain our findings.

Several other potential explanations for our findings should be considered. First, the quality of handoffs may have been sufficient to overcome the potential for information loss. We feel this is unlikely given that little attention had been dedicated to improving the quality of patient handoffs among hospitalists in our institution. Notably, though a number of studies have evaluated resident physician handoffs, most of the work has focused on night coverage, and little is known about the quality of attending handoffs. ${ }^{20}$ Second, access to a fully integrated electronic health record may have assisted hospitalists in complementing information received during handoffs. For 
example, a hospitalist about to start his or her rotation may have remotely accessed and reviewed patient medical records prior to receiving the phone handoff from the outgoing hospitalist. Third, other efforts to improve patient safety may have reduced the overall risk and provided some resilience in the system. Unitbased interventions, including structured interdisciplinary rounds and nurse-physician coleadership, improved teamwork climate and reduced AEs in the study hospital over time. ${ }^{7}$

Another factor to consider relates to the fact that hospital care is provided by teams of clinicians (eg, nurses, specialist physicians, therapists, social workers). Hospital teams are often large and have dynamic team membership. Similar to hospitalists, nurses, physician specialists, and other team members handoff care throughout the course of a patient's hospital stay. Yet, discontinuity for each professional type may occur at different times and frequencies. For example, a patient may be handed off from one hospitalist to another, yet the care continues with the same cardiologist or nurse. Future research should better characterize hospital team complexity (eg, size, relationships among members) and dynamics (eg, continuity for various professional types) and the impact of these factors on patient outcomes.

Our findings are important because hospitalist physician discontinuity is common during hospital stays. Hospital medicine groups vary in their staffing and scheduling models. Policies related to admission distribution and rotation length (consecutive days worked) systematically impact physician continuity. Few studies have evaluated the effect on continuity on hospitalized patient outcomes, and no prior research, to our knowledge, has explored the association of continuity on measures of patient safety. ${ }^{6,21,22}$ Though our study might suggest that staffing models have little impact on patient safety, as previously mentioned, other team factors may influence patient outcomes.

Our study has several limitations. First, we assessed the impact of continuity on AEs in a single site. Although the 7 days on/7 days off model is the most common scheduling pattern used by adult hospital medicine groups, ${ }^{23}$ staffing models and patient safety practices vary across hospitals, potentially limiting the generalizability of our study. Second, continuity can be defined and measured in a variety of ways. We used 2 different measures of physician continuity. As previously mentioned, assessing continuity of other clinicians may allow for a more complete understanding of the potential problems related to fragmentation of care. Third, this study excluded patients who experienced care transitions from other hospitals or other units within the hospital. Patients transferred from other hospitals are often complex, severely ill, and may be at higher risk for loss of key clinical information. Fourth, we used automated screens of an EDW to identify potential AEs. Although our prior research found that this method identified a similar number of AEs as manual medical record review screening, there was poor agreement between the 2 methods. Unfortunately, there is no gold standard to identify AEs. The EDW-facilitated method allowed us to feasibly screen a larger number of charts, increasing statistical power, and minimized any potential bias that might occur during a manual review to identify potential AEs. Finally, we used data available from 2 prior studies and may have been underpowered to detect a significant association between continuity and AEs due to the relatively low percentage of patients experiencing an AE. In a post hoc power calculation, we estimated that we had $70 \%$ power to detect a $33 \%$ change in the proportion of patients with 1 or more AE for each 1 -unit increase in NPI, and $80 \%$ power to detect a $20 \%$ change for each 0.1-unit decrease in UPC.

\section{CONCLUSION}

In conclusion, we found that hospitalist physician continuity was not associated with the incidence of AEs. We speculate that hospitalist continuity is only 1 of many team factors that may influence patient safety, and that prior efforts within our institution may have reduced our ability to detect an association. Future research should better characterize hospital team complexity and dynamics and the impact of these factors on patient outcomes.

Disclosures: This project was supported by a grant from the Agency for Healthcare Research and Quality and an Excellence in Academic Medicine Award, administered by Northwestern Memorial Hospital. The authors report no conflicts of interest.

\section{References}

1. Gulliford M, Naithani S, Morgan M. What is "continuity of care"? J Health Serv Res Policy. 2006;11:248-250.

2. Saultz JW, Lochner J. Interpersonal continuity of care and care outcomes: a critical review. Ann Fam Med. 2005;3:159-166.

3. van Walraven C, Oake N, Jennings A, Forster AJ. The association between continuity of care and outcomes: a systematic and critical review. J Eval Clin Pract. 2010;16:947-956.

4. Saultz JW, Albedaiwi W. Interpersonal continuity of care and patient satisfaction: a critical review. Ann Fam Med. 2004;2:445-451.

5. Blankfield RP, Kelly RB, Alemagno SA, King CM. Continuity of care in a family practice residency program. Impact on physician satisfaction. J Fam Pract. 1990;31:69-73.

6. Turner J, Hansen L, Hinami K, et al. The impact of hospitalist discontinuity on hospital cost, readmissions, and patient satisfaction. J Gen Intern Med. 2014;29:1004-1008.

7. O'Leary KJ, Creden AJ, Slade ME, et al. Implementation of unit-based interventions to improve teamwork and patient safety on a medical service [published online ahead of print June 11, 2014]. Am J Med Qual. doi: 10.1177/1062860614538093.

8. Steinwachs DM. Measuring provider continuity in ambulatory care: an assessment of alternative approaches. Med Care. 1979;17:551565.

9. Saultz JW. Defining and measuring interpersonal continuity of care. Ann Fam Med. 2003;1:134-143.

10. U.S. Department of Health and Human Services. Agency for Healthcare Research and Quality. Adverse events in hospitals: national incidence among medical beneficiaries. Available at: http://psnet.ahrq. gov/resource.aspx? resourceID=19811. Published November 2010. Accessed on December 15, 2014.

11. Classen DC, Resar R, Griffin F, et al. "Global trigger tool” shows that adverse events in hospitals may be ten times greater than previously measured. Health Aff (Millwood). 2011;30:581-589.

12. Hiatt HH, Barnes BA, Brennan TA, et al. A study of medical injury and medical malpractice. N Engl J Med. 1989;321:480-484. 
13. Thomas EJ, Studdert DM, Burstin HR, et al. Incidence and types of adverse events and negligent care in Utah and Colorado. Med Care. 2000;38:261-271.

14. O'Leary KJ, Devisetty VK, Patel AR, et al. Comparison of traditional trigger tool to data warehouse based screening for identifying hospital adverse events. BMJ Qual Saf. 2013;22:130-138.

15. Brennan TA, Leape LL, Laird NM, et al. Incidence of adverse events and negligence in hospitalized patients. Results of the Harvard Medical Practice Study I. N Engl J Med. 1991;324:370-376.

16. Stelfox HT, Bates DW, Redelmeier DA. Safety of patients isolated for infection control. JAMA. 2003;290:1899-1905.

17. HCUP Clinical Classification Software. Agency for Healthcare Research and Quality, Rockville, MD. http://www.hcup-us.ahrq.gov/ toolssoftware/ccs/ccs.jsp. Accessed on December 15, 2014.
18. Elixhauser A, Steiner C, Harris DR, Coffey RM. Comorbidity measures for use with administrative data. Med Care. 1998;36:8-27.

19. Wachter RM. Does continuity of care matter? No: discontinuity can improve patient care. West J Med. 2001;175:5.

20. Arora VM, Manjarrez E, Dressler DD, Basaviah P, Halasyamani L, Kripalani S. Hospitalist handoffs: a systematic review and task force recommendations. J Hosp Med. 2009;4:433-440.

21. Epstein K, Juarez E, Epstein A, Loya K, Singer A. The impact of fragmentation of hospitalist care on length of stay. J Hosp Med. 2010;5: 335-338.

22. Chandra S, Wright SM, Howell EE. The Creating Incentives and Continuity Leading to Efficiency staffing model: a quality improvement initiative in hospital medicine. Mayo Clin Proc. 2012;87:364-371.

23. Society of Hospital Medicine. 2014 state of hospital medicine report. Philadelphia, PA: Society of Hospital Medicine; 2014. 